\title{
Social assistance and climate resilience in fragile and conflict- affected settings
}

\author{
Lars Otto Naess, Jan Selby, Gabrielle Daoust and Jeremy Lind
}

\author{
February 2022
}

\section{Theme summary}

Climate change poses increasing, and changing, risks to people and livelihoods. Fragile and conflict-affected settings (FCAS) host some of the world's most vulnerable populations. Over recent years, social assistance - such as cash transfers and voucher programmes - has been seen as a way of reducing the impacts of climate-related shocks and stressors, and of increasing the resilience of recipient households and communities. It has also been seen as a mechanism for delivering adaptation funding, showing promise in tackling short-term shocks as well as longer-term adaptation to climate change.

Programmes to support better integration of social assistance, adaptation, and resilience have been piloted in countries such as Ethiopia, Tanzania, and India. Using existing and adjusted social assistance efforts to support climate resilience goes to the heart of the COP26 agenda. However, many questions also remain over the suitability and evidence for linkages, and how and under what conditions social assistance interventions may successfully strengthen climate resiliience, and for whom.

This brief gives an overview of the evidence, gaps and future directions for social assistance and climate resilience, with a particular focus on FCAS. Drawing on a recent literature review, we examine existing debates and consider that climate-related social assistance in FCAS involves particular challenges, including risks of maladaptive support.

This thematic brief is a shortened version of a BASIC Research Working Paper. To explore this research theme in more detail please refer to:

Naess, L.O.; Selby, J. and Daoust, G. (2022) Climate Resilience and Social Assistance in Fragile and Conflict-Affected Settings, BASIC Research Working Paper 2, Brighton: Institute of Development Studies, DOI: 10.19088/BASIC.2022.002

A full list of the references cited in this brief can be found at the BASIC Research Zotero library.

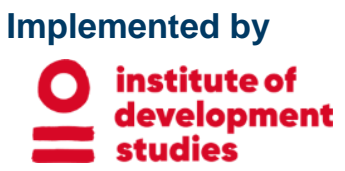

Better Assistance in Crises (BASIC) Research (funded by UKAid) aims to inform policy and programming on how to help poor and vulnerable people cope better with crises and meet their basic needs through more effective social assistance. All costs related to BASIC Research are covered by the UK Foreign, Commonwealth and Development Office. 


\section{State of the evidence and debate}

Social assistance can help climate resilience in four different ways. It can build anticipatory capacity, by improving the lives and livelihoods of the poor against risks, like climate-related shocks and stressors, before they happen. It can help to build absorptive capacity - enabling them to cope with shocks, such as floods, when they happen. It can improve households' longer-term adaptive capacity, by helping to diversify, build assets, or improve understanding of climate information. Last and most challenging, it may improve transformative capacity through addressing deeper, structural drivers - for example, access to land, markets, basic services, social equity, and gender relations.

The literature overwhelmingly focuses on stable contexts, with very little detail on social assistance for climate resilience in FCAS. Four lessons emerge:

- The framing of social assistance-climate change links matters. How climate change is understood and 'framed' has implications for the role that social assistance can play - i.e., knowing which of the capacities are supported. Notably, vulnerability tends to be understood in terms of the effects of biophysical shocks plus social vulnerabilities at the individual, household, and community levels.

- Short-term responses trump longer-term adaptation concerns. There is extensive evidence and agreement that social assistance can contribute to anticipating and absorbing climate-related shocks. The evidence and emphasis on building longer-term adaptive capacities through social assistance is much less than for shock-responsive efforts.

- Risks of maladaptation is an increasing concern. Some existing literature recognises that social assistance programmes may lead to negative coping strategies or maladaptation. Some studies found that when long-term impacts of climate change are not considered in design and planning phases, social assistance may create incentives to stay and invest in locations and livelihoods that may become unviable in the future under increased climate risks.

- Implementing capacities are key. Widespread concern is expressed both within research and policy reports that governments may lack the capacities necessary for integrating climate resilience into social assistance programmes, especially as this may require institutional changes. Challenges identified relate to different forms of technical expertise in the climate change and social protection spheres, different concepts and terminologies, and different government structures and approaches. 


\section{Gaps in the evidence}

We sought to map out some of the challenges in designing and providing social assistance for climate resilience in fragile and conflict-affected situations.

- A lack of focus on longer-term implications of climate vulnerability. Longer-term trends, the implications of these for livelihoods pathways, or needs for adaptation receive less attention than short-term shocks and stressors. As yet, there is insufficient focus on how to ensure that social assistance promotes flexibility and robustness amid uncertainty, and how to design and implement it to avoid the risk of maladaptation. Responses to short-term shocks will not automatically lead to long-term resilience. On the contrary, decisions taken in the short term will affect the prospects of longer-term transformative change.

- A lack of research on underlying drivers and their implications for social assistance. Understanding is still weak regarding the underlying political, economic and environmental causes of vulnerability, including gender inequities and social difference, and the implications of this for social assistance programmes. Most programmes aim to strengthen coping and adaptive capacities within the structural contexts, without addressing the underlying conditions that make people vulnerable. Evidence is scarce to show that social assistance can contribute to transforming the structural causes of vulnerability and building long-term resilience to climate change.

- Missing focus on the specific challenges posed by FCAS for climate and livelihood-promotion related social assistance. This relates both to the causes of climate-related vulnerability in these contexts and the unique difficulties associated with delivery there. These challenges include the extent and gravity of assistance needs; the protracted nature of crises; high incidence or threat of direct political and social violence; weak or distorted legal and institutional regimes affecting state and local capacities to deliver reliable services; disproportionate dependence on humanitarian agencies to jumpstart 'development', and the complexities of local state-international actor relations. 


\section{Directions for research}

Key questions for the next phase of thematic work on climate resilience and livelihoods in BASIC Research:

- What are the politics of vulnerability-social assistance linkages in FCAS? This research will aim to bring together political economy perspectives with assessments of recent and current trends in climate shocks and stressors. The objective is to understand how vulnerabilities arise, are experienced, and negotiated by different social groups living in conditions of conflict, forced (and protracted) displacement, and recurrent climate shocks - and what this implies for social assistance. The starting point is that vulnerabilities are characterised not only by interactions between different types of stressors, but also by the ways that social difference (including processes of inclusion and exclusion) drives exposure to stressors and strategies to cope with them.

- What are desired livelihood pathways, and how can they be made climate resilient in FCAS? The objective here is to examine the potential for social assistance programmes to enhance the resilience of livelihood pathways in the context of protracted crisis. Its starting point will be livelihood pathways, as understood in the case study contexts, and the role of social assistance programmes (especially cash-plus), to build and strengthen resilience to the range of possible, albeit uncertain, future climates.

- What are the programmatic and financial challenges? A cross-cutting concern will be to understand policy implementation, coordination, and financing at national and sub-national levels in FCAS, focused on the nexus of social assistance, humanitarian assistance and climate change. Climate finance may bring potential but also challenges in combination with social assistance to strengthen livelihood resilience and support adaptation. Challenges include poor coordination across developmenthumanitarian assistance, lack of transparency of climate finance support, and lack of involvement of local partners in decision-making or targeting according to local needs and priorities.

\section{Acknowledgements and Disclaimer}

This document was developed by the Better Assistance in Crises (BASIC) Research programme. BASIC is implemented by the Institute of Development Studies (IDS), the University of Sussex and the Centre for International Development and Training, funded by UKAid from the UK government. The views expressed in this document are entirely those of the authors and do not necessarily represent views or policies of the UK governments official policies.

(C) IDS copyright 2022. Copyright in the typographical arrangement and design rests with IDS.

This publication (excluding the logos) may be reproduced free of charge in any format or medium, provided that it is reproduced accurately and not used in a misleading context. The material must be acknowledged as IDS copyright with the title and source of the publication specified.

Published by IDS.

DOI: $10.19088 / B A S I C .2022 .027$ 